\title{
DESIGNAÇÃO ROBUSTA DE GERADORES COM RESTRIÇÕES DE SEGURANÇA PARA GERAÇÃO VOLÁTIL DE ENERGIA EÓLICA
}

\author{
Marcos Roberto Bombacini \\ Universidade Tecnológica Federal do Paraná \\ bombacini@utfpr.edu.br \\ Ademir Alves Ribeiro \\ Universidade Federal do Paraná \\ ademir.ribeiro@ufpr.br
}

\begin{abstract}
Resumo
Atualmente, tanto os operadores nacionais dos sistemas elétricos bem como as concessionárias de energia tem enfrentado e vão enfrentar novos desafios a medida que há um progressivo aumento da geração de energia através de recursos variáveis introduzindose níveis mais elevados de incerteza. O problema da designação de geradores no gerenciamento de energia almeja encontrar o agendamento ótimo minimizando os custos de produção para um conjunto de diferentes unidades de geração: hidrelétricas, termelétricas, eólicas, solares, biogás, etc..., ao mesmo tempo em que satisfaz uma ampla variedade de restrições do sistema. A solução deste problema se traduz em quais geradores serão ligados ou desligados e quanto tempo permanecerão ou mudarão seus estados de acordo com a variação da demanda em tempo real. Novas abordagens para a modelagem são necessárias para resolver este problema com incertezas tais como: estocástica, robusta, restrição de chance. A otimização robusta permite a modelagem de um conjunto de incertezas e garante que a solução encontrada pode satisfazer qualquer escalonamento possível com base nessa incerteza definida. Esta trabalho irá fornecer uma avaliação quanto ao potencial da otimização robusta em desempenhar um papel de tomada de decisão deste problema crucial e diário no setor de energia e como otimização robusta pode ser utilizada para melhorar a confiabilidade do sistema e sua eficiência.

Palavras-Chaves: Otimização Robusta; Designação de Geradores; Incerteza; Programação Matemática.
\end{abstract}

\begin{abstract}
Currently, both national operators of electrical systems as well as power utilities have faced and will face new challenges as there is a progressive increase in power generation using variable resources by introducing higher levels of uncertainty. The issue of the unit commitment in power management aims to find the optimal schedule minimizing production costs for a set of different generation units: hydroelectric, thermoelectric, wind, solar, biogas, etc ... at the same time satisfies a wide variety of system constraints. The solution of this problem translates into which generators will be switched on or off and how long will remain or change their states according to demand change in real time. New approaches to modeling are needed to solve this problem with uncertainties such as stochastic, robust, chanceconstraint. The robust optimization allows modeling a set of uncertainties and ensures that the solution can meet any possible scaling based on this defined uncertainty. This work will provide an assessment of the potential of robust optimization play a decision-making role of this crucial and daily problem in energy and how robust optimization industry can be used to improve system reliability and efficiency.

Keywords: $\quad$ Robust Optimization; Unit Commitment; Uncertainty; Mathematical Programming.
\end{abstract}




\section{INTRODUÇÃO}

Inicialmente o problema de designação de geradores foi modelado sem considerar a integração da energia eólica. Posteriormente foi considerada a introdução da energia eólica no sistema elétrico. No primeiro modelo, o problema foi formulado como um problema de otimização determinística. Nesta formulação, o escalonamento era feito para cada cenário de produção eólica e no final determinava-se a melhor solução por meio de indicadores de avaliação. No segundo modelo, o problema foi formulado como um problema de otimização estocástico. Nesta formulação todos os cenários de produção eólica eram levados em consideração no processo de otimização.

Foram feitas simulações para diferentes níveis de reserva girante e os resultados obtidos mostraram que a alta participação da energia eólica na matriz energética põe em risco a segurança e a garantia de produção devido as característica voláteis e intermitentes e para manter os mesmos níveis de segurança é preciso dispor no sistema de capacidade reserva girante suficiente capaz de compensar os erros de previsão.

O problema da designação de geradores no gerenciamento de energia almeja encontrar o agendamento ótimo de produção para um conjunto de unidades de geração ao mesmo tempo em que uma ampla variedade de restrições do sistema é satisfeita. Este sempre foi um problema difícil, de larga escala e não convexo, especialmente tendo em vista o fato de que os requisitos operacionais implicam que o mesmo precisa ser resolvido em um tempo "injustificavelmente" pequeno; recentemente, a capacidade de geração sempre crescente das energias renováveis tem contribuído fortemente para o nível de incerteza no sistema, tornando o modelo de designação de geradores ideal um programa de larga escala, não convexo, incerto, estocástico, robusto, com restrição de chance.

Antes de tratar da inclusão das incertezas no modelo de designação de geradores, são destacados alguns dos trabalhos mais relevantes sobre o problema da designação de geradores na seção 2. A seção 3 expõe as incertezas presentes no problema de designação de geradores e inclui a formulação determinística base do problema. A seção 4 apresenta a formulação que será utilizada para a implementação e para os testes computacionais numéricos. Finalmente, os resultados esperados são destacados na seção 5.

\section{O PROBLEMA DA DESIGNAÇÃO DE GERADORES}

O agendamento ótimo em curto prazo de geradores para atender a demanda de energia elétrica com um baixo risco tem sido motivo de preocupação das concessionárias de energia nas últimas cinco décadas e por esta razão isto tem sido amplamente tratado pela literatura [1], [2], [3], [4], [5]. Este problema é conhecido por Designação de Geradores, Unit Commitment, em inglês. Um modelo detalhado de designação de geradores corresponde a um problema em larga escala de programação não linear inteira mista, onde o custo operacional de todos os geradores, sujeito ao equilíbrio de potência e as restrições técnicas em cada intervalo do período de otimização, é minimizado. Entretanto, com o passar do tempo diferentes modelos aproximados foram usados e métodos intuitivos tais como Enumeração Exaustiva [6] e Lista Prioritária [7] foram inicialmente aplicados a pequenos sistemas elétricos de potência. Mais tarde, o aumento simultâneo da infraestrutura elétrica e do poderio computacional levaram à aplicação de técnicas formais de otimização tal como a abordagem da Programação Dinâmica [8] para resolver o problema da designação de geradores. Desde as primeiras aplicações da Programação Dinâmica, a formulação matemática do problema da designação não parou de evoluir e várias revisões bibliográficas foram publicadas. Algumas delas serão discutidas para obter uma progressão histórica [1], [2], [3], [4], [5]. Até o ano 2000 duas principais tendências da evolução puderam ser identificadas: modelagem de novas restrições operacionais e aplicação de diferentes técnicas de otimização.

Em 1987, Cohen et al. estudaram 76 referências e discutiram o escopo do problema da designação de geradores em curto prazo, com ênfase nas restrições hidro térmicas [1]. Os 
métodos de solução disponíveis naquela época para o problema do escalonamento de geradores eram: Programação Dinâmica, Relaxação Lagrangeana, Método Branch-and-Bound e decomposição Benders. Em 1994, Sheble et al. apresentaram uma lista de 80 referências disponíveis para a solução do problema do escalonamento de geradores termelétricos e concluíram que a Relaxação Lagrangeana era a técnica mais promissora para resolver o problema de designação de geradores em curto prazo [2]. Os inconvenientes para as abordagens da Programação Dinâmica foram o tratamento sub ótimo das restrições dos geradores intertemporais e a necessidade de limitar as designações consideradas em cada hora para envolver a natureza combinatória do problema da designação de geradores. Além disso, o princípio da otimalidade pode ser violado quando as restrições de tempo mínimo ligado/desligado são consideradas [9].

Em 2004, Padhy reuniu 35 anos de pesquisas da designação de geradores incluindo cerca de 150 artigos publicados [3]. Três tópicos foram discutidos: o primeiro foi minimização do custo da designação de geradores versus as primeiras formulações de maximização de lucro, a segunda foi a inclusão das restrições em relação à rede e as restrições de segurança na designação de geradores e o terceiro foi as técnicas de otimização. Naquela época uma nova tendência foi identificada em relação a uma abordagem de otimização mais adequada para problemas de designação de geradores: os modelos híbridos baseados em uma combinação de técnicas consideradas clássicas e das técnicas mais inovadoras empregando métodos heurísticos. O artigo de Padhy foi atualizado em 2012 por Bhardwaj et al. [4], mas comentários com relação a tendências para pesquisa foram evitadas. Finalmente, um resumo de literatura exaustivo, com mais de 400 referências, em métodos de otimização aplicados a designação de geradores em larga escala, com atenção especial dada para considerações sobre incerteza, foi publicado por Tahanan et al. [5].

Atualmente, os sistemas de potência veem enfrentando transformações operacionais devido à integração da geração variável de energias renováveis. A variabilidade adicional e a incerteza deste tipo de geração tem aberto uma nova linha de pesquisa relacionando a sua incorporação a demanda da geração ao problema da designação de geradores. Este acréscimo adicional na geração com característica variável incide sobre a capacidade do sistema de potência preservando economicamente o equilíbrio de energia sem o aumento do risco operacional. O uso do critério clássico de segurança na formulação do escalonamento de geradores, em particular o requisito de reserva determinístico $\mathrm{N}-1$, tem sido questionado em publicações científicas, tanto por razões econômicas quanto por razões de segurança [10]. Assim, diferentes alternativas têm sido propostas, principalmente nos últimos 10 anos, com a ênfase recente na representação explícita das incertezas dentro do modelo de designação de geradores.

Neste sentido, um consenso em favor das formulações da Programação Linear Inteira Mista parece emergir. Argumentos para esta escolha têm sido apresentados na literatura, mas uma compreensiva discussão nas técnicas de otimização aplicadas na designação de geradores sob incertezas poderia ser prematuro, por exemplo, as abordagens da Otimização Robusta e da Otimização com Restrição de Chances ainda precisam ser exploradas com maior profundidade. Alternativamente, uma visão geral sob as considerações da incerteza em modelos da designação de geradores poderia auxiliar os pesquisadores e a indústria a manter a rota dos desenvolvimentos recentes no tópico. O objetivo deste trabalho não é fornecer uma revisão de literatura exaustiva, mas apresentar a evolução dos modelos de designação de geradores com uma apresentação de certos aspectos que impactam na segurança do sistema. 


\section{GERENCIAMENTO DE INCERTEZAS NO PROBLEMA DE DESIGNAÇÃO DE GERADORES}

Uma tendência particularmente relevante nos sistemas elétricos atuais é sempre aumentar o uso de fontes de produção renováveis ou intermitentes tais como a energia eólica e a energia solar. Esta prática tem aumentado significativamente a incerteza implícita no sistema, anteriormente quase completamente devida à demanda dos usuários, cuja previsão poderia ser realizada de maneira bastante eficiente, e da ocorrência de falhas, que poderia ser considerando requerendo alguma quantidade de reserva girante. Ignorar tal acréscimo substancial nos níveis de incerteza em relação aos modelos comuns existentes incorre em um risco inaceitável que o cálculo do agendamento da produção pode ser significativamente mais oneroso do que se fosse antecipado, ou mesmo inviável. Entretanto, incorporar a incerteza nos modelos é bastante desafiador, em particular em vista das dificuldades nas versões determinísticas da designação de geradores.

Felizmente, os métodos de otimização capazes de lidar com incerteza têm sido uma área de pesquisa bastante ativa na última década, e vários desses desenvolvimentos podem e têm sido aplicados ao problema da designação de geradores. Isto é facilmente explicado, além da importância prática da designação de geradores com incerteza, pela combinação de dois fatores: por um lado a diversidade de ambientes operacionais que precisam ser levados em consideração, e por outro lado o fato de que as múltiplas técnicas de solução aplicáveis já disponíveis para a designação de geradores ser muito mais formatada pela necessidade de decidir como a incerteza será modelada. Na verdade, a literatura oferece três tipos de abordagens que possuem requisitos práticos e computacionais substancialmente diferentes: Otimização Estocástica, Otimização Robusta e Otimização com Restrição de Chance. A escolha de uma ou de outra modelagem possui enormes implicações no formato da designação de geradores com incerteza, sua potencial robustez a despeito da incerteza, o custo esperado dos agendamentos calculados da produção e o custo computacional de determinálos; tudo isto implica que a designação de geradores com incerteza ainda não está tão bem resolvido quanto a designação determinística de geradores também sendo uma área de

\subsection{UMA VISÃO GERAL DA DESIGNAÇÃO DE GERADORES}

Em termos gerais, o problema da designação de geradores determinística ou com incertezas requer minimizar o custo, ou maximizar o lucro obtidos pelo agendamento da produção para as unidades geradoras no decorrer de um dado horizonte de tempo. Como tal, os ingredientes fundamentais da designação de geradores são sua função objetivo e suas restrições. Obviamente, outro ingrediente fundamental é o próprio horizonte de planejamento, a designação de geradores sendo um modelo em curto prazo ocorre mais frequentemente nas operações de um ou dois dias, e de até uma semana. A seguir será denotado por $T$, que é tipicamente considerado ser um conjunto discreto correspondendo a um número finito de instantes de tempo $t \in T$, geralmente horas e meia horas dividido em intervalos de quinze ou de cinco minutos; assim, o tamanho típico de $T$ varia de 24 a poucas centenas.

Em termos matemáticos, a designação de geradores tem uma estrutura geral:

$$
\min \left\{f(x): x \in X_{1} \cap X_{2},\right\}
$$

$x \in R^{n}$ é o vetor de tomada de decisão. Normalmente a maioria dos elementos de $x$ são indexados tanto com a unidade geradora $i=1, \ldots, m$ quanto com o instante de tempo $t \in T$ a que eles se referem; assim pode-se falar dos sub-vetores $x^{t}$ como sendo todas as decisões 
pertencentes ao tempo $t$ e/ou $x_{i}$ como sendo todas as decisões pertencentes ao gerador $i$. Ainda, as entradas para $x$ são tipicamente divididas entre:

1. decisão de designação, variáveis discretas que determinam se um gerador em particular está ligado ou desligado em um dado tempo, geralmente denotado por $u_{i}^{t}$;

2. decisão de produção, variáveis contínuas que determinam a quantidade de energia gerada por um gerador específico em um dado tempo, geralmente denotado por $p_{i}^{t}$;

3. decisão da rede, tais como aquelas representando o ângulo de fase ou a magnitudes das tensões, descrevendo o estado da rede de transmissão ou de distribuição.

\subsection{MÉTODOS DE OTIMIZAÇÃO ROBUSTA PARA DESIGNAÇÃO DE GERADORES COM INCERTEZA}

As abordagens de otimização robusta levam a custos substancialmente mais elevados para o escalonamento proposto, um preço para a robustez muito alto [10] com relação as abordagens da otimização estocástica quando as distribuições da incerteza são suficientemente bem caracterizadas. Isto se deve principalmente porque a otimização robusta protege-se contra cada evento no conjunto de incerteza especificado independentemente de sua probabilidade, e, portanto pode considerar eventos extremamente improváveis, embora várias abordagens de otimização robusta tenham parâmetros, ou seja, orçamentos para incerteza, que podem ser usados para ajustar os graus de proteção oferecidos pelo modelo [11], [12], [13], mesmo assim, em geral ajustar esses parâmetros é bastante trivial. Para reduzir o preço da robustez associado com os clássicos conjuntos elipsoidais e os conjuntos incertos com $\Gamma$-robustez propostos em [14], [10], [15], estudos subsequentes investigaram modelos robustos suaves e leves [16], [17]. Mais recentemente, Robustez Multibanda [18], [19], foi proposto como uma generalização dos $\Gamma$-robustez que podem suportar uma representação estratificada e melhorada da incerteza e uma redução no seu conservadorismo, enquanto mantém a tratabilidade computacional e a acessibilidade do $\Gamma$-robustez.

O mais antigo trabalho utilizando as técnicas da otimização robusta foi [20], onde um problema claro de mercado foi considerado sob algumas restrições do tipo da designação de geradores. A principal ideia foi usar uma abordagem de otimização robusta adaptativa que particiona o conjunto incerto e permite que as decisões sejam especificadas para cada subconjunto; as restrições são então ponderadas no problema principal. Os resultados são comparados com a otimização robusta tradicional e a abordagem completa anteciparia o pior caso.

Em [21] uma abordagem de otimização robusta foi considerada onde o conjunto incerto da carga foi um simples intervalo, de maneira que os métodos a partir do intervalo da programação linear, por exemplo [22], podem ser empregados junto com a decomposição Benders para resolver o modelo. O principal enfoque do trabalho está na segurança da rede.

Em [23] uma abordagem de intervalo de incerteza semelhante foi comparada com a abordagem baseada em cenários; os resultados mostraram que a primeira é bastante sensível para a escolha do intervalo, mas é rapidamente resolvida, enquanto que a segunda abordagem produz soluções mais precisas mais é mais onerosa para ser resolvida.

Em [24] um escalonamento básico de 36 geradores com restrições de taxa de rampa foi considerado incluindo a alimentação com energia eólica e o comportamento da demanda 
dos consumidores baseado nos preços da eletricidade. Neste modelo de dois estágios, a energia eólica entrou sob o aspecto de uma restrição orçamentária com incerteza e o primeiro estágio foi um problema de designação de geradores futuro, enquanto o segundo estágio foi realizado uma vez que a alimentação eólica seja conhecida. O problema foi resolvido aplicando decomposição Benders para o problema linearizado junto com um algoritmo em linguagem de programação C. Afirmou-se que este modelo reduziu significativamente o custo total e pode explorar completamente a fonte disponível de energia eólica. A mesma abordagem foi empregada em [25] para resolver um escalonamento com 30 geradores com restrições de taxa de rampa e de transmissão onde demanda e oferta sendo consideradas como incertezas.

Em [26] o modelo proposto em [25], [24] foi estendido e incorporou as restrições de reserva girante, limites de transmissão e restrições de rampa para ajustar o impacto da robustez das soluções na eficiência e na estabilidade operacional do sistema. Um modelo de otimização robusta adaptativo de dois estágios foi usado onde o conjunto incerto atendeu a injeção nodal na rede em cada período de tempo. No primeiro estágio uma decisão de designação ótima foi alcançada pelo algoritmo de decomposição Benders, enquanto que no segundo estágio o custo de despacho de pior caso foi calculado. Os resultados a partir de estudos empíricos com 312 geradores foram comparados a aqueles dos modelos determinísticos com ajustes de reserva sob três aspectos: o despacho médio e o custo total, a volatilidade do custo, e a sensibilidade dos custos para diferentes distribuições de probabilidade. A sensibilidade dos resultados a mudanças no conjunto incerto não foi investigada.

Em [27] uma abordagem de otimização robusta para o gerenciamento de geração de energia elétrica foi apresentado usando conceitos adaptados do gerenciamento de risco clássico, ou seja, análise de risco. Em [28] uma otimização robusta com a abordagem de homólogo robusto ajustável afim [29] foi proposto para um gerenciamento em um prazo mais longo da produção de eletricidade; a AARC é uma versão restrita e mais tratável do homólogo robusto ajustável onde as variáveis de decisão são passíveis de depender dos valores passados dos parâmetros incertos, mas apenas de uma maneira afim. Finalmente, em [30] um ótimo fluxo de potência robusto ajustável foi sugerido.

\section{DESIGNAÇÃO DE GERADORES COM RESTRIÇÕES DE SEGURANÇA EÓLICA}

\subsection{NOMENCLATURA}

$i, k, t, w$ : índices dos geradores termelétricos, segmentos curvos, horas, fazendas eólicas

$O R_{i t}, S R_{i t}$ : reservas operacionais, reservas girantes do gerador $i$ no tempo $t$

$P_{i k t}^{b}$ : despacho do gerador $i$ do segmento $k$ no tempo $t$ no caso base

$P_{D t}^{u}, P_{f, w t}^{u}$ : carga do sistema com incerteza, energia eólica com incerteza para a

fazenda eólica $w$ no tempo $t$ no interior de intervalo com incerteza

$S U_{i t}, S D_{i t}$ : custo de partida, custo de parada do gerador $i$ no tempo $t$

$X_{o n, i t}, X_{o f f, i t}$ : tempo ligado do gerador, tempo desligado do gerador $i$ no tempo $t$

$c_{i k}$ : custo incremental para o segmento $k$ do gerador $i$

$O R_{t}, S R_{t}$ : limites das reservas operacionais, reservas girantes no tempo $t$ 
$P_{D t}, P_{f, w t}$ : carga do sistema, energia eólica prevista para a fazenda eólica $w$ no tempo $t$

$P_{D t}^{-}, P_{D t}^{+}$: limite inferior, limite superior do intervalo de incerteza na carga para o intervalo de tempo $t$

$P_{f, w t}^{-}, P_{f, w t}^{+}$: limite inferior, limite superior do intervalo de incerteza de vento para a fazenda eólica $w$ no intervalo de tempo $t$

$P_{i}^{\min }, P_{i}^{\max }$ : capacidade mínima, capacidade máxima do gerador $i$

$P_{i k}^{\max }$ : capacidade de potência do segmento $k$ do gerador $i$

$P L_{l}^{\max }$ : limite superior para o fluxo de potência da linha $l$

$R_{i}^{u p}, R_{i}^{\text {down }}$ : limite superior, limite inferior da ação corretiva no gerador $i$

$S F_{l, m}$ : fator de deslocamento da linha $l$ e do barramento $m$

$s u_{i}, s d_{i}$ : custo de partida, custo de parada do gerador $i$

$T_{o n, i}, T_{o f f, i}$ : limite de tempo mínimo ligado, limite de tempo mínimo desligado do gerador $i$

$U R_{i}, D R_{i}$ : limite de taxa de subida, limite de taxa de descida de rampa do gerador $i$

$D(m)$ : conjunto de todas as demandas de carga localizadas no barramento $m$

$U(m)$ : conjunto de todos os geradores localizados no barramento $m$

$W(m)$ : conjunto de todas as fazendas eólicas localizadas no barramento $m$

\subsection{FORMULAÇÃO DETERMINÍSTICA DA DESIGNAÇÃO DE GERADORES COM RESTRIÇÕES DE SEGURANÇA}

A designação de geradores com restrições de segurança determinística tem sido extensivamente estudada na literatura como um problema de programação linear inteira mista [31], [32]. O objetivo da designação de geradores com restrições de segurança é o de minimizar os custos totais de operação, incluindo o custo de produção de energia, o custo sem carga, e o custo de partida/parada ao longo de um horizonte de agendamento inteiro (2). As restrições predominantes na designação de geradores com restrições de segurança incluem equilíbrio da carga do sistema (3), limites da capacidade de geração de geradores termelétricos (4), (5), (6), limites na geração das fazendas eólicas (7), limites de tempo mínimo em que um determinado gerador permanece ligado ou desligado (8), (9), restrições de custo para a partida ou para a parada de um determinado gerador (10), (11), limites para as rampas de subida e de descida (12), (13). As restrições de limites para reserva girante, para a reserva operacional, e para o combustível, para a emissão e a restrição de segurança para a rede de transmissão (14) fazem cumprir os limites de capacidade das linhas de transmissão. Os custos variáveis de tempo para partida e para a parada de geradores também podem ser incorporados ao modelo proposto. A formulação detalhada também pode ser encontrada em trabalhos anteriores [33].

$$
\operatorname{Min} \sum_{t} \sum_{i}\left(\sum_{k} c_{i k} \cdot P_{i k t}^{b}+N_{i} \cdot I_{i t}^{b}+S U_{i t}+S D_{i t}\right)
$$

Para cada gerador $i$, o valor de $c_{i k}$ está aumentando para valores maiores de $k$ para garantir que a função de custo seja convexa. Dominando as restrições da designação de geradores com restrições de segurança incluem o equilíbrio de carga do sistema:

$$
\sum_{i} P_{i t}^{b}+\sum_{w} P_{w t}^{b}=\sum_{d} P_{d t}^{b}
$$


os limites da capacidade de geração dos geradores termelétricos:

$$
\begin{gathered}
P_{i}^{\min } \cdot I_{i t}^{b} \leq P_{i t}^{b} \leq P_{i}^{\max } \cdot I_{i t}^{b} \\
0 \leq P_{i k t}^{b} \leq P_{i k}^{\max } \cdot I_{i t}^{b} \\
P_{i t}^{b}=\sum_{k} P_{i k t}^{b}
\end{gathered}
$$

os limites na geração das fazendas eólicas:

$$
0 \leq P_{w t}^{b} \leq P_{f, w t}^{b}
$$

limites de tempo mínimo em que um determinado gerador permanece ligado ou desligado:

$$
\begin{gathered}
\left.\left[X_{o n, i(t-1)}-T_{o n, i}\right] \cdot \mid I_{i(t-1)}^{b}-I_{i t}^{b}\right\rfloor \geq 0 \\
{\left[X_{o f f, i(t-1)}-T_{o f f, i}\right] \cdot\left|I_{i t}^{b}-I_{i(t-1)}^{b}\right| \geq 0}
\end{gathered}
$$

restrições de custo para a partida ou para a parada de um determinado gerador:

$$
\begin{array}{ll}
S U_{i t}^{b} \geq S u_{i} \cdot\left(I_{i t}^{b}-I_{i(t-1)}^{b}\right), & S U_{i t}^{b} \geq 0 \\
S D_{i t}^{b} \geq S d_{i} \cdot\left(I_{i(t-1)}^{b}-I_{i t}^{b}\right), & S D_{i t}^{b} \geq 0
\end{array}
$$

limites para as rampas de subida e de descida:

$$
\begin{aligned}
& P_{i t}^{b}-P_{i(t-1)}^{b} \leq U R_{i} \cdot I_{i(t-1)}^{b}+P_{i}^{\min } \cdot\left(I_{i t}^{b}-I_{i(t-1)}^{b}\right)+P_{i}^{\max } \cdot\left(1-I_{i t}^{b}\right) \\
& P_{i(t-1)}^{b}-P_{i t}^{b} \leq D R_{i} \cdot I_{i t}^{b}+P_{i}^{\min } \cdot\left(I_{i(t-1)}^{b}-I_{i t}^{b}\right)+P_{i}^{\max } \cdot\left(1-I_{i(t-1)}^{b}\right)
\end{aligned}
$$

as restrições de limites para reserva girante, para a reserva operacional, e para o combustível, para a emissão e a restrição de segurança para a rede de transmissão:

$$
-P L_{l}^{\max } \leq \sum_{m} S F_{l, m}\left(\sum_{i \in U(m)} P_{i t}^{b}+\sum_{w \in W(m)} P_{w t}^{b}-\sum_{d \in D(m)} P_{d t}^{b}\right) \leq P L_{l}^{\max }
$$

\subsection{FORMULAÇÃO ROBUSTA DA DESIGNAÇÃO DE GERADORES COM RESTRIÇÕES DE SEGURANÇA}

Os valores previstos para a demanda de carga do sistema $P_{d t}^{b}$ e a geração eólica $P_{f, w t}^{b}$ no modelo determinístico para designação de geradores (2)-(14) pode ser impreciso. Neste trabalho, $P_{D t}^{u} \in\left[P_{D t}^{-}, P_{D t}^{+}\right]$e $P_{f, w t}^{u} \in\left[P_{f, w t}^{-}, P_{f, w t}^{+}\right\rfloor$são utilizados para representar conjuntos de possíveis cargas e possíveis realizações de energia eólica. Isto é, realizações da demanda de carga do sistema e realizações da geração eólica poderiam assumir quaisquer valores no interior dos seus intervalos de incerteza. $\mathrm{O}$ modelo robusto de designação de geradores com restrições de segurança é formulado como a seguir. O objetivo (15) é o de minimizar os custos 
totais de operação do caso base com relação às restrições (2)-(14) mais as restrições a seguir para tratar incertezas.

As restrições para tratar os intervalos de incerteza incluem os limites de geração dos geradores termelétricos (17) e as fazendas eólicas (18). Ajustes no despacho dos geradores termelétricos em resposta aos intervalos de incerteza estão restritos pelas suas capacidades de ação corretivas e a solução de despacho no caso base, que garante que o estado da operação do sistema possa ser seguramente transferido a partir do seu caso base para todas as possíveis realizações eólicas e de carga no interior de seus intervalos de incerteza (20), (21). Restrições de transmissão da rede (22) são obrigadas as serem cumpridas para garantir a segurança da rede quando o vento e a carga estiverem variando no interior de seus intervalos de incerteza. $P L_{t}^{u}\left(P_{D t}^{u}, P_{f, w t}^{u}\right), P_{i t}^{u}\left(P_{D t}^{u}, P_{f, w t}^{u}\right)$ e $P_{w t}^{u}\left(P_{D t}^{u}, P_{f, w t}^{u}\right)$ são conjuntos variáveis que representam todos os possíveis fluxos de potência e valores de despacho em resposta a diferentes realizações de carga e de vento no interior dos seus intervalos de incerteza.

$$
\underset{P_{i k}^{b}, P_{i t}^{b}, I_{i t}^{b}, P_{w t}^{b}}{\sum_{t}} \sum_{i}\left(\sum_{k} c_{i k} \cdot P_{i k t}^{b}+N_{i} \cdot I_{i t}^{b}+S U_{i t}+S D_{i t}\right)
$$

A restrição de equilíbrio do sistema:

$$
\sum_{i} P_{i t}^{u}\left(P_{d t}^{u}, P_{f, w t}^{u}\right)+\sum_{w} P_{w t}^{u}\left(P_{d t}^{u}, P_{f, w t}^{u}\right)=\sum_{d} P_{d t}^{u}
$$

garante o equilíbrio de carga no sistema quando o vento e a carga assumirem quaisquer valores no interior de seus intervalos de incerteza. As equações a seguir descrevem os limites da geração dos geradores termelétricos e das fazendas eólicas sob incertezas:

$$
\begin{gathered}
P_{i}^{\mathrm{min}} \cdot I_{i t}^{b} \leq P_{i t}^{u}\left(P_{d t}^{u}, P_{f, w t}^{u}\right) \leq P_{i}^{\max } \cdot I_{i t}^{b} \\
0 \leq P_{w t}^{u}\left(P_{d t}^{u}, P_{f, w t}^{u}\right) \leq P_{f, w t}^{u}
\end{gathered}
$$

Os ajustes no despacho dos geradores termelétricos em resposta aos conjuntos incertos estão restritos por sua capacidade corretiva e aos despachos de geração no caso base:

$$
-R_{i}^{\text {down }} \cdot I_{i t}^{b} \leq P_{i t}^{u}\left(P_{d t}^{u}, P_{f, w t}^{u}\right)-P_{i t}^{b} \leq R_{i}^{u p} \cdot I_{i t}^{b}
$$

As equações a seguir representam os limites de rampa de subida e de descida dos geradores termelétricos sob os conjuntos incertos:

$$
\begin{aligned}
& P_{i t}^{u}\left(P_{d t}^{u}, P_{f, w t}^{u}\right)-P_{i(t-1)}^{u}\left(P_{d(t-1)}^{u}, P_{f, w(t-1)}^{u}\right) \leq U R_{i} \cdot I_{i(t-1)}^{b}+P_{i}^{\min } \cdot\left(I_{i t}^{b}-I_{i(t-1)}^{b}\right)+P_{i}^{\max } \cdot\left(1-I_{i t}^{b}\right) \\
& P_{i(t-1)}^{u}\left(P_{d(t-1)}^{u}, P_{f, w(t-1)}^{u}\right)-P_{i t}^{u}\left(P_{d t}^{u}, P_{f, w t}^{u}\right) \leq D R_{i} \cdot I_{i t}^{b}+P_{i}^{\min } \cdot\left(I_{i(t-1)}^{b}-I_{i t}^{b}\right)+P_{i}^{\max } \cdot\left(1-I_{i(t-1)}^{b}\right)
\end{aligned}
$$

Diferentes de outros conjuntos incertos que possuem $N T$ restrições correspondendo a cada tempo $t,(20)-(21)$ possui apenas $(N T-1)$ restrições onde a faixa de $t$ varia entre 2 a $N T$. A restrição da rede de transmissão 


$$
-P L_{l}^{\max } \leq\left[\sum_{m} S F_{l, m} \cdot\left(\sum_{i \in U(m)} P_{i t}^{u}\left(P_{i t}^{u}, P_{f, w t}^{u}\right)+\sum_{w \in W(m)} P_{w t}^{u}\left(P_{d t}^{u}, P_{f, w t}^{u}\right)-\sum_{d \in D(m)} P_{d t}^{u}\right)\right] \leq P L_{l}^{\max }
$$

garante a segurança da rede sob incertezas.

\section{RESULTADOS ESPERADOS}

Os impactos de vários fatores de incerteza na segurança e na operação econômica de sistemas de potência são críticas, especialmente quanto mais e mais recursos renováveis voláteis e a resposta a demanda são integradas aos sistemas de potência existentes. Espera-se efetuar a implementação do modelo descrito na seção 4 no software MATLAB ${ }^{\circledR}$ e obter resultados numéricos mais eficientes a partir do modelo robusto proposto. A metodologia se mostra promissora para a solução do problema da designação de geradores com restrições de segurança minimizando o caso base para os valores previstos, ao mesmo tempo em que cobre a maior parte das realizações incertas no interior de seus intervalos de incerteza ajustando de maneira adaptativa ajustando o despacho das unidades geradoras. Enquanto a designação de geradores com restrições de segurança determinística não pode ser considerado atualmente um problema totalmente resolvido, o surgimento da injeção de energia nos sistemas de potência existentes advindo das energias renováveis insere incertezas que necessitam ser incorporadas no modelo.

\section{REFERÊNCIAS BIBLIOGRÁFICAS}

[1] COHEN, A. I.; SHERKAT, V. R. Optimization-Based Methods for Operating Scheduling. Proceedings of the IEEE, v. 75, n. 12, p. 1574-1591.

[2] SHEBLE, G. B., AND FAHD, G. N. Unit Commitment Literature Synopsis. IEEE Transactions on Power Systems, v. 9, n. 1, p. 128-135, 1994.

[3] PADHY, N. P. Unit Commitment - A Bibliographical Survey. IEEE Transactions on Power Systems, v. 19, n. 2, p. 1196-1205, 2004.

[4] BHARDWAJ, A., KAMBOJ, V. K., SHUKLA, V. K., SINGH, B., AND KHURANA, P. Unit Commitment in Electrical Power System - A Literature Review. IEEE International Power Engineering and Optimization Conference. Anais... p.6-7, 2012.

[5] TAHANAN, M, ACKOOIJ, W. V., FRANGIONI, A., LACALANDRA, F. Largescale Unit Commitment Under Uncertainty : A Literature Survey. Pisa, 2014.

[6] KERR, R. H., SCHEIDT, J. L., FONTANA, JR, A. J., AND WILEY, J. K. Unit Commitment. IEEE Transactions on Power Apparatus and Systems, v. PAS-85, n. 5, p. 417-421, 1966.

[7] LEE, F. Short-Term Thermal Unit Commitment - A New Method. IEEE Transactions on Power Systems, v. 3, n. 2, p. 421-428, 1988.

[8] SNYDER, W. L., POWELL, H. D., and RAYBURN, J. C. Dynamic Programming Approach to Unit Commitment. IEEE Transactions on Power Systems, v. PWRS-2, n. 2, p. 339-348, 1987.

[9] GOOI, H. B., MENDES, D. P., BELL, K. R. W., KIRSCHEN, D. S. Optimal Scheduling of Spinning Reserve. IEEE Transactions on Power Systems, v. 14, n. 4, p. 1485-1492, 1999. 
[10] BERTSIMAS, D. AND SIM, M. The Price of Robustness. Operations Research, v. 52, n. 1, p. 35-53, 2004.

[11] BERTSIMAS, D. AND SIM, M. Robust discrete optimization and network flows. Mathematical Programming, v. 98, p. 49-71, 2003.

[12] CHEN, X., SIM, M., AND SUN, P. A Robust Optimization Perspective on Stochastic Programming. Operations Research, v. 55, n. 6, p. 1058-1071, 2007.

[13] NEMIROVSKI, A., AND SHAPIRO, A. Convex Approximations of Chance Constrained Programs. SIAM Journal on Optimization, v. 17, n. 4, p. 969-996, 2006.

[14] BEN-TAL, A AND NEMIROVSKI, A. ROBUST CONVEX OPTIMIZATION. Mathematics of Operations Research, v. 33, p. 769-805, 1998.

[15] GHAOUI, L. E., OUSTRY, F., AND LEBRET, H. Robust solutions to uncertain semidefinite programs. SIAM Journal on Optimization, v. 9, p. 33-52, 1998.

[16] BEN-TAL, A., BERTSIMAS, D., BROWN, D. B. A Soft Robust Model for Optimization Under Ambiguity. Operations Research, v. 58, n. 4, p. 1220-1234, 2010.

[17] FISCHETTI, M., AND MONACI, M. Light Robustness. Robust and Online LargeScale Optimization, v. 58, n. 1, p. 1-21, 2009.

[18] BÜSING, C., and D'ANDREAGIOVANNI, F. New results about multi-band uncertainty in robust optimization, Springer Berlin Heidelberg, 2012.

[19] BÜSING, C., and D'ANDREAGIOVANNI, F. Robust Optimization under Multi-band Uncertainty Part I: Theory, Technical Report, Zuse-Institut Berlin, 2013.

[20] SARIC, A. T., AND STANKOVIC, A. M. Finitely Adaptive Linear Programming in Robust Power System Optimization. Power Tech. Anais... . p.1302-1307, 2007.

[21] WANG, Y., XIA, Q., AND KANG, C. Unit Commitment With Injections by Using Interval Optimization. IEEE Transactions on Power Systems, v. 26, n. 3, p. 17051713, 2011.

[22] CHINNECK, J. W., and RAMADAN, K. Linear Programming with Interval Coefficients. The Journal of the Operational Research Society, v.51, n. 2, p. 209220, 2000.

[23] WU, L., SHAHIDEHPOUR, M., AND LI, Z. Comparison of Scenario-Based and Interval Optimization Approaches to Stochastic SCUC. IEEE Transactions on Power Systems, v. 27, n. 2, p. 913-921, 2012.

[24] ZHAO, L., AND ZENG, B. Robust Unit Commitment Problem with Demand Response and Wind Energy. Power and Energy Society General Meeting. Anais... . p.1-8, 2012.

[25] JIANG, R., ZHANG, M., LI, G., AND GUAN, Y. Two-Stage Robust Power Grid Optimization Problem. 2010.

[26] BERTSIMAS, D., LITVINOV, E., SUN, X. A., ZHAO, JI. AND ZHENG, T. Adaptive Robust Optimization for the Security Constrained Unit Commitment Problem. IEEE Transactions on Power Systems, v. 28, n. 1, p. 52-63, 2013.

[27] AID, R., GUIGUES, V., NDIAVE, P.-M., OUSTRY, F., and ROMANET, F. A ValueAt-Risk approach for robust management of electricity power generation. Technical Report, 2006.

[28] GUIGUES, V. Robust Product Management. Optimization and Engineering, v. 10, n. 4, p. 505-532, 2009. 
[29] BEN-TAL, A., GORYASHKO, A., GUSLITZER, E., and NEMIROVSKI, A. Adjustable Robust Counterpart of Uncertain Linear Programs. Mathematical Programming, v. 99, p. 351-376, 2003.

[30] JABR, R. A. Adjustable Robust OPF With Renewable Energy Sources. IEEE Transactions on Power Systems, v. 28, n. 4, p. 4742-4751, 2013.

[31] GUAN, X., ZHAI, Q., PAPALEXOPOULOS, A. Optimization Based Methods for Unit Commitment: Lagrangian Relaxation versus General Mixed Integer Programming. IEEE Power Engineering Society General Meeting. Anais... . p.1095-1100, 2003.

[32] LI, T., SHAHIDEHPOUR, M. Price-Based Unit Commitment : A Case of Lagrangian Relaxation Versus Mixed Integer Programming. IEEE Transactions on Power Systems, v. 20, n. 4, p. 2015-2025, 2005.

[33] WU, L., SHAHIDEHPOUR, M., AND LI, T. Stochastic Security-Constrained Unit Commitment. IEEE Transactions on Power Systems, v. 22, n. 2, p. 800-811, 2007. 